\title{
Protector effect of esmolol on thoracic aorta remodeling by ADMA pathway in arterial hypertension
}

\author{
Martín-Oropesa R., Gómez B., Ávalos E., Ramos E., García-Reza A., Quintana-Villamandos B. \\ Hospital General Universitario Gregorio Marañón, Dept of Anaesthesiology, Madrid, Spain
}

\section{Background and Goal of Study:}

Our group previously demonstrated that short-term treatment $(48 \mathrm{~h})$ with esmolol reduces left ventricular

hypertrophy by increasing the bioavailability of nitric oxide (1), although the impact of short-term treatment with this beta-blocker on remodeling in large arteries has not yet been studied. We hypothesized that even a short ( $48 \mathrm{~h}$ ) course of esmolol might alter remodeling of the aorta in the spontaneously hypertensive rat (SHR).

\section{Materials and Methods:}

Fourteen-month-old male SHRs were treated intravenously with vehicle $(\mathrm{SHR}, \mathrm{n}=8)$ or esmolol (SHR-E, $\mathrm{n}=8)$. Age-matched, vehicle-treated male Wistar-Kyoto rats (WKY, $\mathrm{n}=8$ ) served as controls. After $48 \mathrm{~h}$, we studied the structure, volume density of elastic fibers, and passive mechanical properties of the aorta (B parameter, wall stiffness). Determination of asymmetrical dimethylarginine (ADMA) concentrations in the aorta was analyzed. The results were expressed as mean \pm S.E.M. The parameters were compared using single-factor (rat) analysis of variance. A post hoc

Bonferroni correction was applied. Non-regression analysis with an exponential equation was used to estimate mechanical parameters (B parameter). $P$-values $<0.05$ were considered statistically significant. All procedures were approved by the Ethics Committee of Hospital General Universitario Gregorio Marañon, Madrid, Spain.
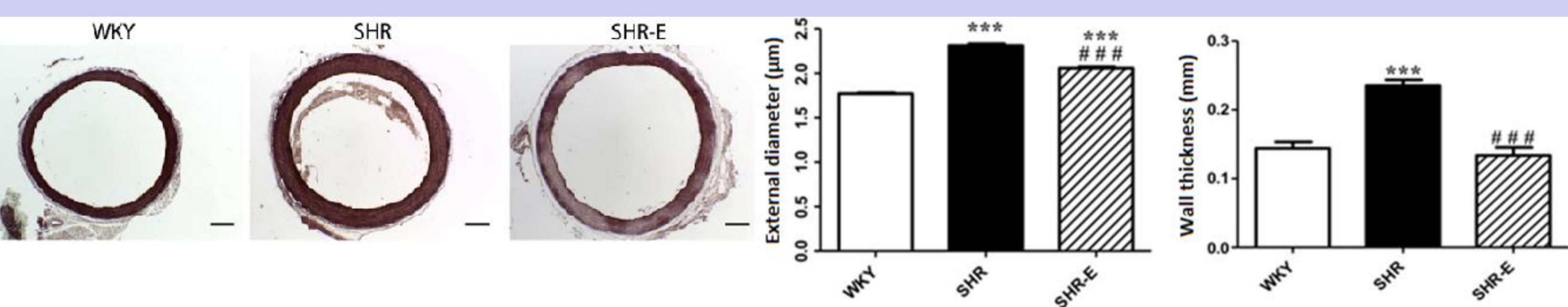

Examples of histological sections of the aorta (Orcein 40x). Structural parameters: external diameter ( $\mu \mathrm{m})$, Wall thickness $(\mathrm{mm})$ in WKY, SHR and SHR-E. Statistically significant differences are shown: ${ }^{\star \star \star} p<0,001$ versus WKY, \#\# $p<0,001$ vesus SHR
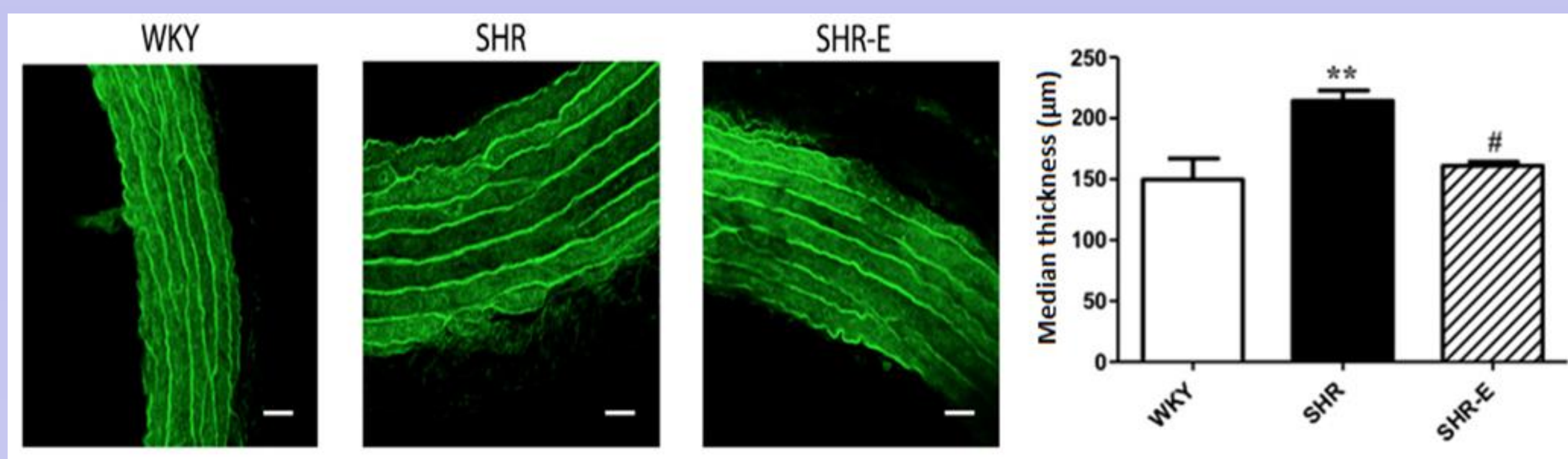

\begin{abstract}
Examples of the tunica media obtained using confocal microscopy (20x). Structural parameters: median thickness $(\mu \mathrm{m})$ in WKY, SHR and SHR-E. Statistically significant differences are shown: ${ }^{\text {** }} p<0,01$ versus WKY $\# p<0,05$ vesus $S H R$
\end{abstract}
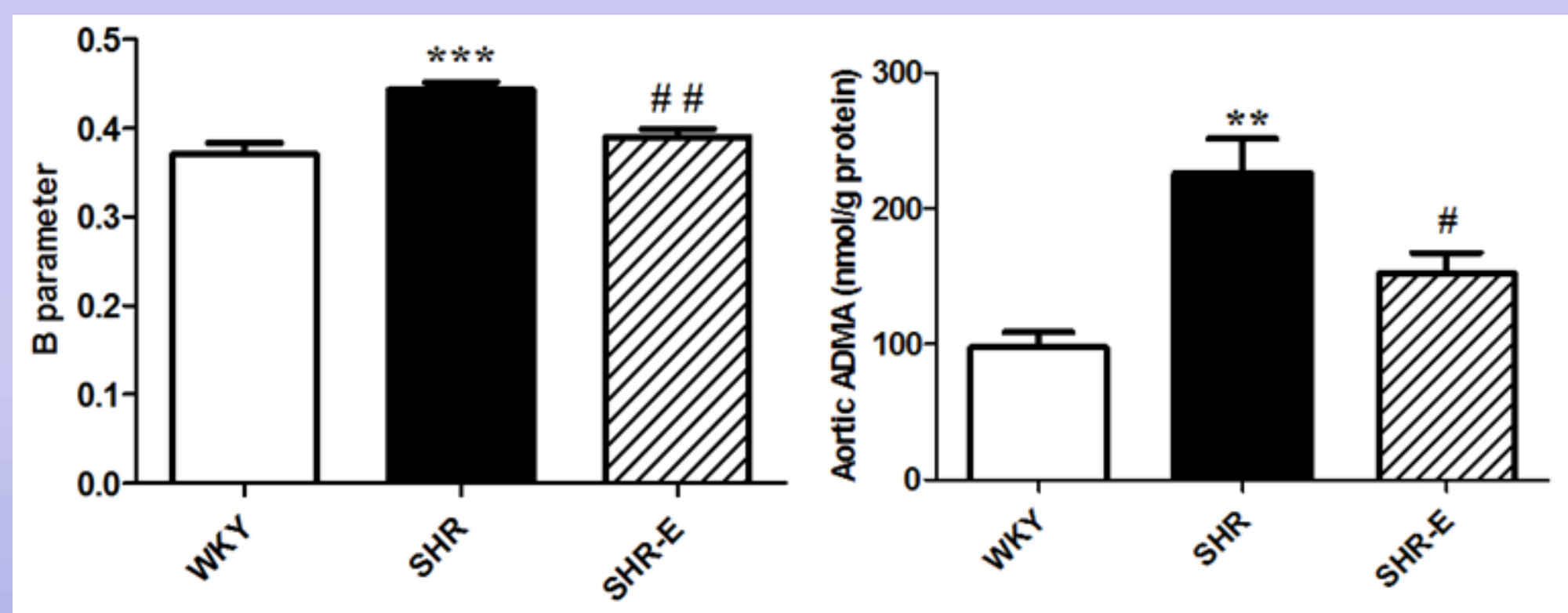

Passive mechanical properties of the aorta (B parameter) and ADMA in WKY, SHR and SHR-E. Statically signidicant differences are shown: ${ }^{* \star} p<0,01$ versus $W K Y,{ }^{* \star *} p<0,001$ versus $W K Y, \# p<0,05$ versus SHR, \#p<0,01 versus SHR.

\section{CONCLUSION:}

Esmolol is now widely used to treat patients with arterial hypertension. These findings could play a key role in the selection of antihypertensive therapy in patients with hypertension and aortic remodeling

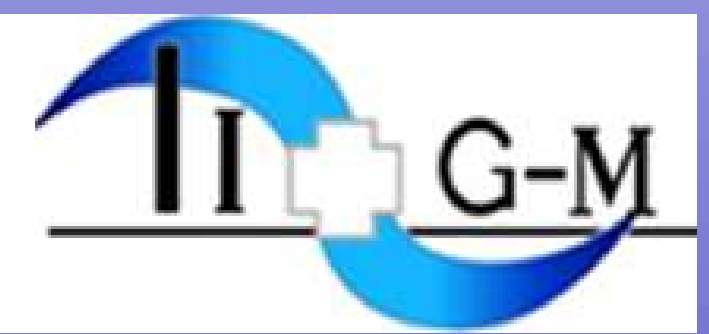

